\title{
TRANSFER CHARACTERISTICS OF FIRST AND SECOND ORDER LATERAL CANAL VESTIBULAR NEURONS IN GERBIL
}

\author{
L. W. SCHNEIDER* and D. J. ANDERSON
}

Kresge Hearing Research Institute, University of Michigan, Medical School, Ann Arbor, Mich. (U.S.A.)

(Accepted January 6th, 1976)

SUMMARY

Discharge patterns of first and second order vestibular neurons responding to angular acceleration in the plane of the lateral canals were studied in gerbil. The resting discharge activity of each cell was used to characterize the neuron by measuring the coefficient of variation and coefficient of skewness of the interspike interval distributions. Sinusoidal angular oscillations ranging in frequency from 0.0125 to 5.0 $\mathrm{Hz}$ were delivered by a velocity controlled rate-table. A PDP-12 minicomputer system was used on-line to display period and post-stimulus histograms of discriminated single unit activity. Off-line Fourier analysis of the period histograms was used to determine the phase of cell response to sinusoidal accelerations, while the average level and amplitude were determined by a least squares fitting algorithm applied over the fraction of the stimulus period where the cell discharged. First order neurons were found to have high discharge rates (average $=61.7 \mathrm{imp} . / \mathrm{sec}$ ) and bidirectional responses to rotation, and were of two groups called regular and irregular according to their resting discharge patterns. Second order neurons, located mainly in the medial and lateral vestibular nuclei, had low or even zero resting discharge rates (average $=$ 17.8) resulting in more uni-directional responses and were of a single population.

For frequencies less than $10 \mathrm{~Hz}$, the Bode plots of the regular first order neurons are similar to that of a first order system with a time constant of about $2 \mathrm{sec}$ as predicted by the torsion pendulum theory for cupula movement. The irregular first order neurons show an increasing gain above $0.5 \mathrm{~Hz}$ and a large phase lead relative to angular velocity above $1.0 \mathrm{~Hz}$ suggestive of a fractional power transfer function. The second order neurons show the phase and gain characteristics of the regular first order neurons being in phase with angular velocity above $1.0 \mathrm{~Hz}$.

* Present address: Highway Safety Research Institute, University of Michigan, Ann Arbor, Mich. 48105, U.S.A. 


\section{INTRODUCTION}

The hypothesis that the cupula-endolymph system in the semicircular canal behaves as an overdamped torsional pendulum has, for many years, been regarded as an accurate description of this receptor mechanism. Since the origin of this model by Steinhaussen in 1931, there have been many experiments which basically substantiate the theory, and the concept of the cupula bending or swinging sluggishly within the membranous ampulla has become popular.

In recent years, however, new evidence has been presented which indicates that modifications to this description may be needed. The results of several studies $8,10,15$, for example, suggest that the cupula displacement or bending is on the order of hundredths or thousandths of a degree rather than many degrees, and therefore, to consider the cupula as actually swinging within the ampulla is inaccurate. In addition, as was shown by Fernández and Goldberg 4 in the squirrel monkey, the transfer characteristics of all first order vestibular neurons cannot be explained on the basis of the torsion pendulum model for cupula movement. Using sinusoidal angular rotations at frequencies between 0.1 and $8.0 \mathrm{~Hz}$, they demonstrated that some first order vestibular neurons (those with irregular resting discharge characteristics) showed, in addition to the phenomenon of adaptation, an increasing phase lead with respect to angular velocity and an increasing sensitivity with increasing stimulus frequency above $1.0 \mathrm{~Hz}$. It was suggested that these lead characteristics may serve to compensate for dynamic loads at other points in the vestibular reflex pathways, and thereby extend the frequency range for which the system can compensate for angular velocity of the animal.

Jones and Milsum ${ }^{8}$ and Shinoda and Yoshida ${ }^{11}$ have studied the transfer characteristics of second order vestibular neurons in the brain stem of cats but neither used stimulation frequencies above $1.0 \mathrm{~Hz}$. In the present study the frequency response characteristics of both first and second order vestibular neurons in gerbils were obtained over the frequency range of $0.01-5.0 \mathrm{~Hz}$ in order to confirm and extend the above results for the gerbil, and compare the dynamic behavior of first and second order vestibular neurons.

\section{METHODS}

Single cell recordings from the vestibular ganglion and vestibular nuclei were obtained from 20 gerbils (Meriones unguiculatus). Animals were anesthetized with a combination of ketamine hydrochloride (Ketalar) (15 mg i.m.) and urethane $(25 \mathrm{mg}$ i.p.). After cannulation of the tracheae the animals were secured by a head holder and placed on a rate table so that the lateral canals were in the plane of table rotation. A cranial opening was made over the cerebellum and the dura reflected. For vestibular nuclei recordings, indium filled micropipettes platinized at the tip were inserted through the cerebellum and into the brain stem. These electrodes are such that they record only from cell bodies and thus insured that only second and higher order neurons were recorded from, at this level. Lesions were made after recording from a 
single cell by passing a $\mathrm{DC}$ current $(30-60 \mu \mathrm{A}$ for $1 \mathrm{~min}$ electrode positive) through the electrode. For recording in Scarpa's ganglion, the cerebellum was first aspirated, exposing the VIIIth cranial nerve where it emerges from the internal auditory canal. Sodium chloride filled micropipettes were then placed into the nerve under visual control with the aid of an operating microscope.

Impulse activity of single cells was amplified by an AC preamplifier (Grass $\mathrm{P}-15$ ) on the turntable and connected via slip rings to monitoring and recording equipment. Output of the preamplifier passed to a dual beam oscilloscope (Tektronix 565), one trace of which was used to discriminate single unit discharge potentials. By triggering this trace with the spike voltage and connecting the gate-out of this trace to one channel of a KW-12 timer associated with a PDP-12 computer (Digital Equipment Corp.), post-stimulus and cycle histograms were generated on-line. All responses were also monitored audibly and stored in analog form using an instrumentation tape recorder (Hewlett Packard, model 3960).

Vestibular cells sensitive to stimulation of the semicircular canals were found by advancing the microelectrode while rotating the turntable by hand. Upon isolating a responding cell, resting discharge activity was recorded for $30-60 \mathrm{sec}$. This was followed by an initial rotation sequence of $32^{\circ}$ or $64^{\circ} / \mathrm{sec}$ amplitude at $0.5 \mathrm{~Hz}$ to determine the cell sensitivity and identify its type. First order neurons were considered to be of lateral canal origin if their discharge rate increased on ipsilateral rotation. Second order neurons were classified as type I lateral canal cells if their discharge rate increased on ipsilateral rotation and type II lateral canal or type I vertical (posterior or anterior) canal cells if their discharge rate decreased on ipsilateral rotation. Only lateral canal first order neurons and type I lateral canal second order neurons were used for frequency response analysis.

Once a cell was identified as a lateral canal first order neuron or a type I lateral canal second order neuron, sinusoidal rotations ranging in frequency from 0.01 to $5.0 \mathrm{~Hz}$ were delivered. For very sensitive cells, stimulus amplitudes were maintained at $32 \% \mathrm{sec}$ throughout the frequency range. For less sensitive cells it was often necessary to increase the stimulus amplitude up to $96^{\circ} / \mathrm{sec}$ at the lower frequencies in order to obtain a sufficient response. If a cell survived the complete frequency spectrum, a series of sinusoidal stimuli at $0.5 \mathrm{~Hz}$ over a range of amplitudes was applied to determine the dependency of cell sensitivity on stimulus amplitude. If the cell was still uninjured and not lost by these stimuli a series of long duration step accelerations (ramp velocities) were given to determine the step response.

The rotational stimuli were delivered by a rate table with velocity feedback control (Inland model TT-5742A), powered by an Inland Controls 60-ICM-600 power supply. Sinusoidal rotations in the horizontal plane were achieved by driving the system with a Hewlett Packard function generator (model 3310A).

Data were digitized off-line by playback of the analog tape, using the oscilloscope to discriminate neural activity and the PDP-12 computer with KW-12 timer to digitize the times of impulse events and stimulus sync marks. These digital data were stored on computer-compatible magnetic tape and were later used to construct poststimulus, interspike-interval, and period histograms. 
The amplitude, phase, and DC level of the cell response to sinusoidal stimuli were determined by Fourier analysis of the period histograms for those cells showing continuous modulation over the cycle. These period histograms were the average steady state response obtained from the series of stimulus rotations at each frequency. For cells which were silenced during a portion of the cycle, the phase of the response was also computed by Fourier analysis, while the amplitudes and average levels were determined by a least squares fitting of a sinusoid to the active (non-zero) portion of the period histogram. For cells which were silent in the resting state, a negative DC level was calculated, providing a measure of the cell hyperpolarization.

Period histograms and computed 'best-fit' curves for these 3 cases of high resting discharge with continuous firing throughout the stimulus cycle, low resting discharge with partial cut-off, and spontaneously silent cell with extensive cut-off and negative DC level are shown in A, B, and C, respectively of Fig. 1. It should be emphasized that the frequency response results computed in this way are in terms of angular velocity of the animal and therefore gain or sensitivity is expressed as impulses/sec of neuron firing per degree/sec of table rotation. Final results are reported in Bode plot form, where gain is in decibels and zero decibels is $1 \mathrm{imp}$./sec per degree/sec.

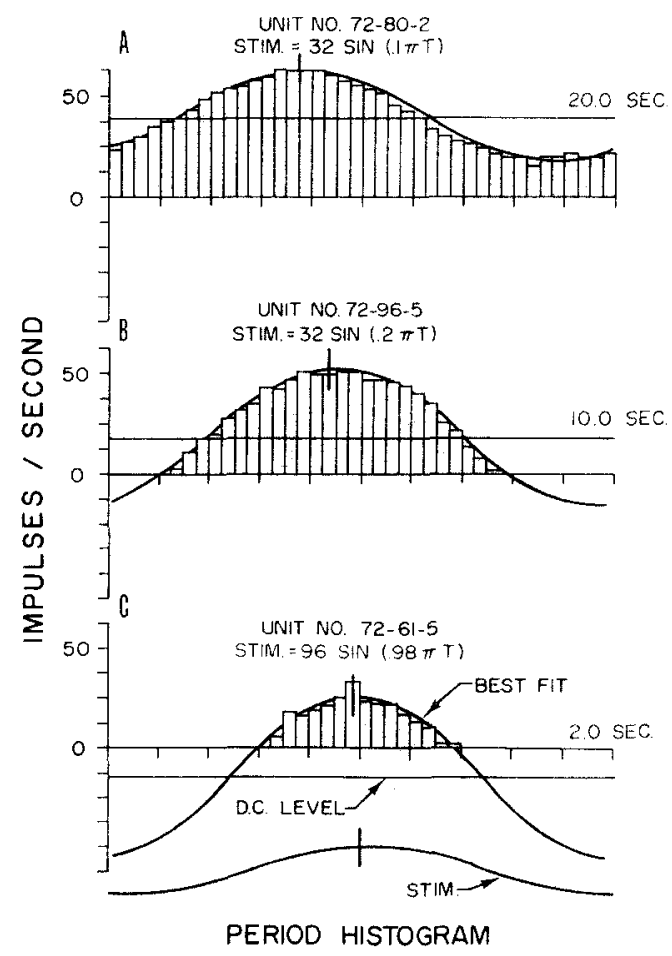

Fig. 1. Period histograms showing response to sinusoidal stimuli of a continuously firing cell in A, a cell with partial cut-off in $B$, and a spontaneously silent cell with extensive cut-off and negative $D C$ level in $\mathrm{C}$. 


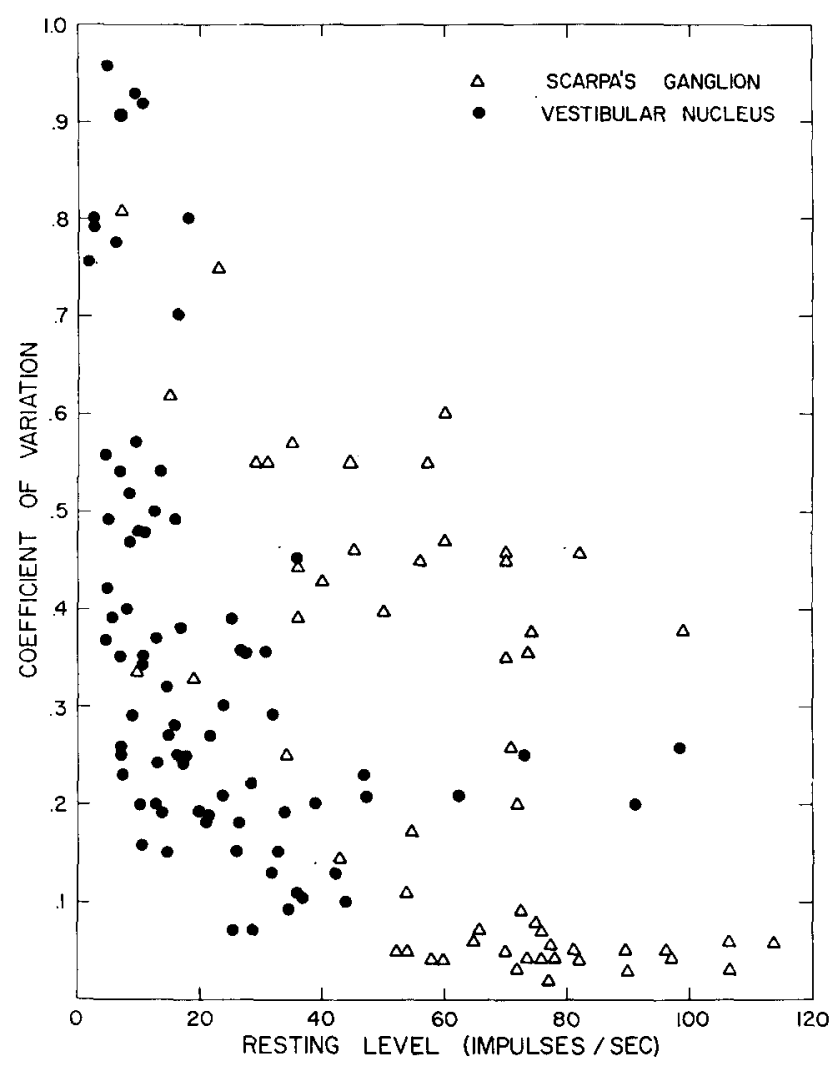

Fig. 2. Relation between coefficient of variation of interval distribution and resting discharge rate for first and second order vestibular neurons.

\section{RESULTS}

\section{Resting discharge characteristics}

The interval histogram describes the distribution of intervals between adjacent impulses in a neural train and therefore is an estimate of the probability function for the occurrence of an impulse from the time of a previous impulse. The shape of this function can be quantified by calculating the mean, coefficient of variation (c.v.), and the coefficient of skewness* (s.k.) of the interval distribution. The number of impulses divided by the total sample time gives the average rate of cell firing, while the coefficient of variation and coefficient of skewness measure the spread and asymmetry of the interval distribution, respectively. These parameters were calculated for the resting discharge patterns recorded and provide a basis for classifying or grouping the cells.

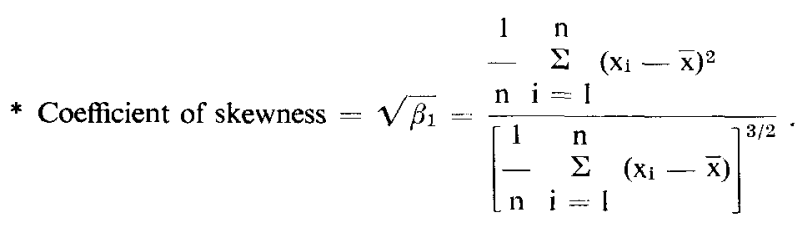


In this study coefficient of variation was chosen to describe the cell regularity. Fig. 2 is a plot of the coefficient of variation versus the resting discharge level for 57 vestibular ganglion cells and 83 vestibular nuclei cells. The figure illustrates the relatively high discharge rates of the first order neurons relative to the second order neurons. For the populations shown, the average resting rate of the Scarpa's ganglion cells is $61.7 \mathrm{imp}$./ $\mathrm{sec}$ (S.D. 25.3) as compared to $17.8 \mathrm{imp} . / \mathrm{sec}$ (S.D. 18.0) for the nuclei cells. In the vestibular nuclei, several 'silent' neurons were also found for which negative resting levels (not shown) were calculated by the curve fitting procedure previously described. As was found by Jones and Milsum ${ }^{8}$, these silent cells fit into and make the distribution of average resting levels of second order cells more normal or Gaussian.

With regard to regularity of the resting discharge pattern, the first order neurons seem to group into two populations of regular (low c.v.) and irregular (high c.v.) while the second order neurons show a unimodal distribution indicative of a single population. Further, the majority of regular first order neurons have lower c.v.'s than any of the second order neurons. While it cannot be disputed that there is a continuous range of discharge patterns for the first order neurons from the very regular to the irregular, the distinct population of low c.v. cells leads to a tendency to classify these neurons into two groups, called regular and irregular. This has been done by others $t$, 13,15 , and is with the understanding that the division between the two is not sharp. For this study, cells are classified as regular if the c.v. is less than or equal to 0.10 and as irregular if the c.v. is greater than 0.10 . The second order cells will be treated is a single population. The results which follow, therefore, are grouped into the 3 categories of first order regular, first order irregular, and second order cells.

\section{Sinusoidal response characteristics}

Sensitivity. The responses of 35 type I lateral canal neurons in the vestibular nuclei, and 26 lateral canal neurons in Scarpa's ganglion to sinusoidal rotation were obtained. For the first order neurons the average sensitivity was $0.58 \mathrm{imp}$. $/ \mathrm{sec}$ per degree/sec (S.D. $=0.35$ ) while for the type I second order cells it was 0.68 (S.D.

0.47 ). Of the 26 first order neurons, 9 were classified as regular and had an average sensitivity of 0.43 (S.D. $=0.28$ ) while 17 were irregular and had an average sensitivity of 0.68 (S.D. $=0.36$ ).

Response linearity. In order for linear system techniques, such as frequency response analysis, to be a valid tool for determining system transfer characteristics, the system under study must be essentially linear. That this is a reasonable assumption for the vestibular system has been shown by others ${ }^{4,15}$. The results of the present study also justify the use of these methods.

Fig. 3 illustrates the change in sensitivity with stimulus amplitude for 3 cells in Scarpa's ganglion and 5 cells in the vestibular nuclei. In general, the gain of a cell is seen to decrease as the stimulus amplitude is increased. This relationship between gain and stimulus amplitude is nearly linear for most cells and the rate of decrease appears to be proportional to the cell sensitivity at low amplitudes. For the two most sensitive second order cells, however, the gain shows an almost logarithmic decrease in sensi- 


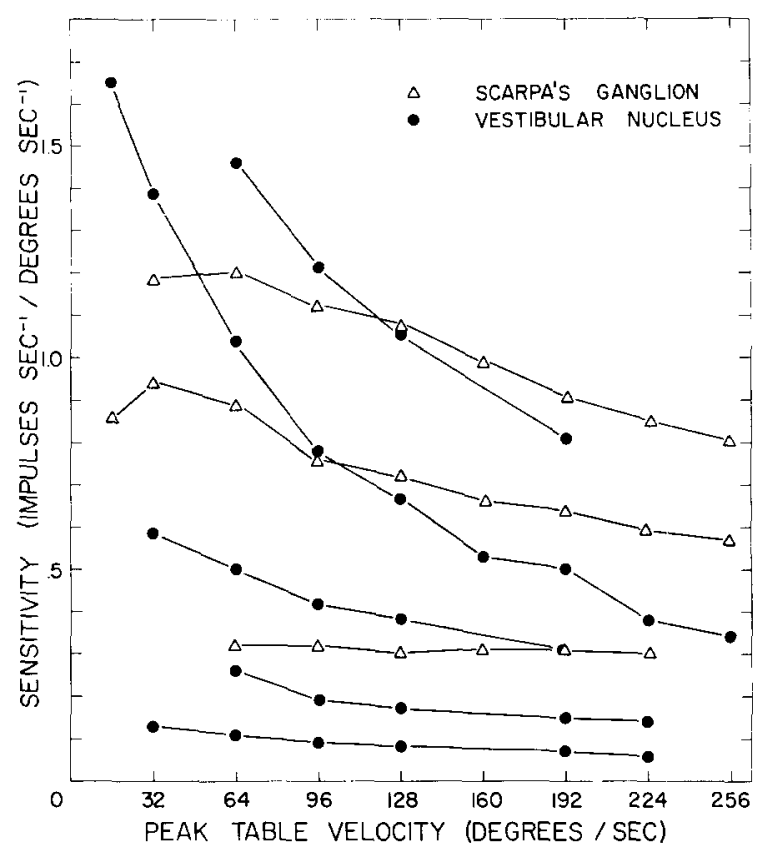

Fig. 3. Relation between cell sensitivity and amplitude of sinusoidal stimulus for 3 cells in Scarpa`s ganglion and 5 cells in the vestibular nuclei.

tivity with increasing stimulus amplitude. In consideration of this non-linearity, the frequency response results of this study were obtained by maintaining the stimulus amplitude constant for very sensitive cells and between $32^{\circ}-64^{\circ} / \mathrm{sec}$ for less sensitive cells for all stimulus frequencies.

With few exceptions, the non-zero portions of the responses to sinusoidal stimulation can be fit by sinusoidal functions with the addition of a bias term over the frequency and amplitude range used in this study. Typical examples of these responses are illustrated in Fig. 1.

An exception to a sinusoidal response histogram occurred in the few vestibular nucleus cells which were rather insensitive, and had a low or zero resting discharge rate. In these instances, the period histogram was skewed as shown in Fig. 4A. The period histograms and dot pattern of Fig. 4B illustrate that this skewness is the result of synchronization of the neural discharge to the phase of the stimulus cycle. This is due to the fact that the cell polarization continues to be modulated with the stimulus during the silent period and passes through threshold at the same time in every cycle. The regularity of the discharge pattern determines the degree to which the first impulse is synchronized to the stimulus cycle. Successive discharges within the active region of the stimulus period become less and less synchronized as the indeterminacy of interspike intervals accumulates. Period histograms of this cell type apear to be modulating with the stimulus as they approach the silent portion of the cycle. If the period of the cycle is short the synchronization appears to be more pronounced. This 

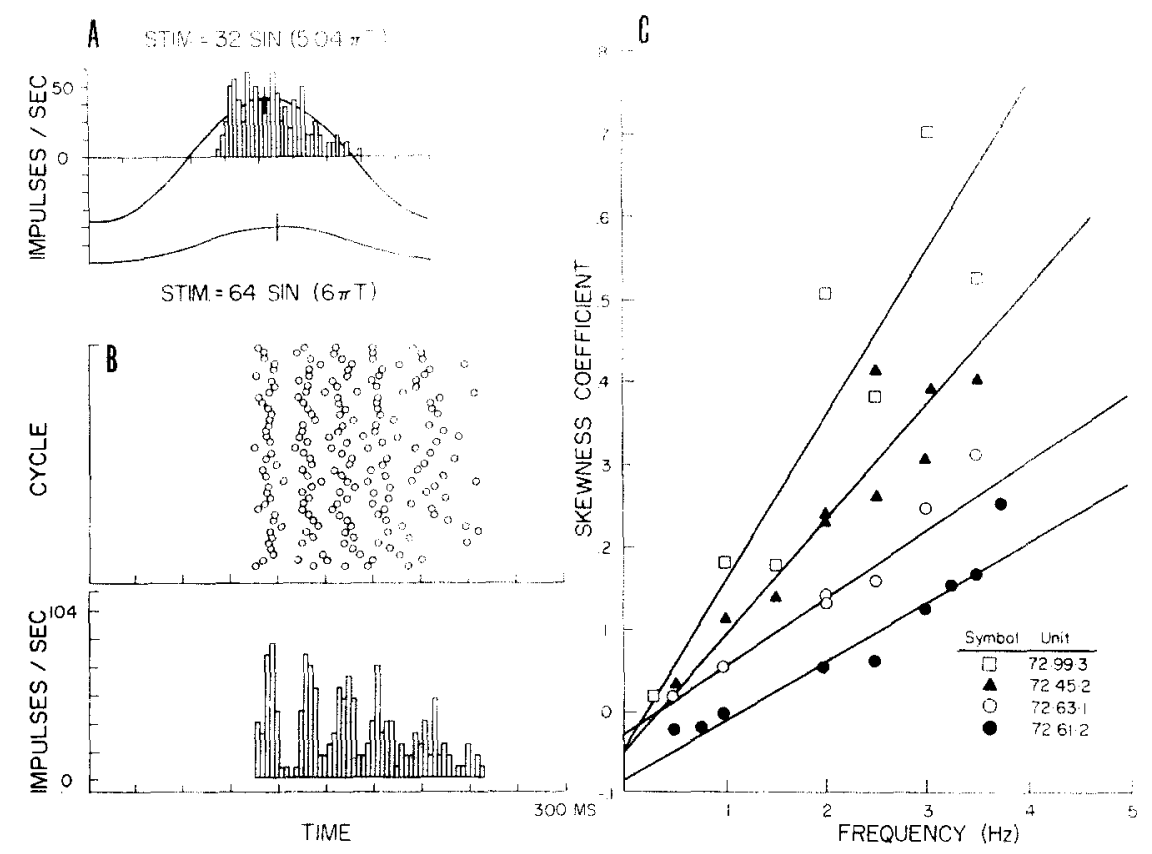

Fig. 4. A: period histogram showing cut-off and resulting skewness; B : dot pattern and corresponding period histogram illustrating synchronization mechanism causing skewness; $\mathrm{C}$ : relation between the coefficient of skewness (Pearson $\beta_{1}$ ) of the period histogram and the frequency of sinusoidal stimulus for 4 cells in the vestibular nuclei. Solid lines are the linear regression fits to the data.

can be demonstrated by calculating the skewness factor as a function of stimulus frequency. Data from 4 such units are illustrated in Fig. $4 \mathrm{C}$.

It should be pointed out that this skewness is largely due to the nature of the period histogram which is the sum of the responses of one cell to many cycles of rotation. Physiologically a neuron at the next level takes its input from an ensemble of many different neurons. Since the resting discharge levels and regularity for these different cells are distributed over a large range, it is unlikely that this skewness or synchronization is physiologically important. The effect upon the frequency response results must, however, be considered. Data from cells exhibiting severe skewness at higher frequencies were not included in the results to follow. Bode plots from some cells exhibiting only moderate skewing were, however, included in the final results for the vestibular nuclei. As can be noted from Fig. 4A, the 'best fit' curve and the active portion of the period histogram agree well at the end of neuron firing, indicating that the phase (which is still that of a fundamental calculated by Fourier analysis) is probably a good measure of the actual cell response. The effect of the skewness, if any, would be to increase the phase lead. The amplitude or gain of the computed response is also probably increased somewhat by the skewing.

Bode plot results

Scarpa's ganglion. Fig. 5A shows the average Bode plot for 8 regular lateral 

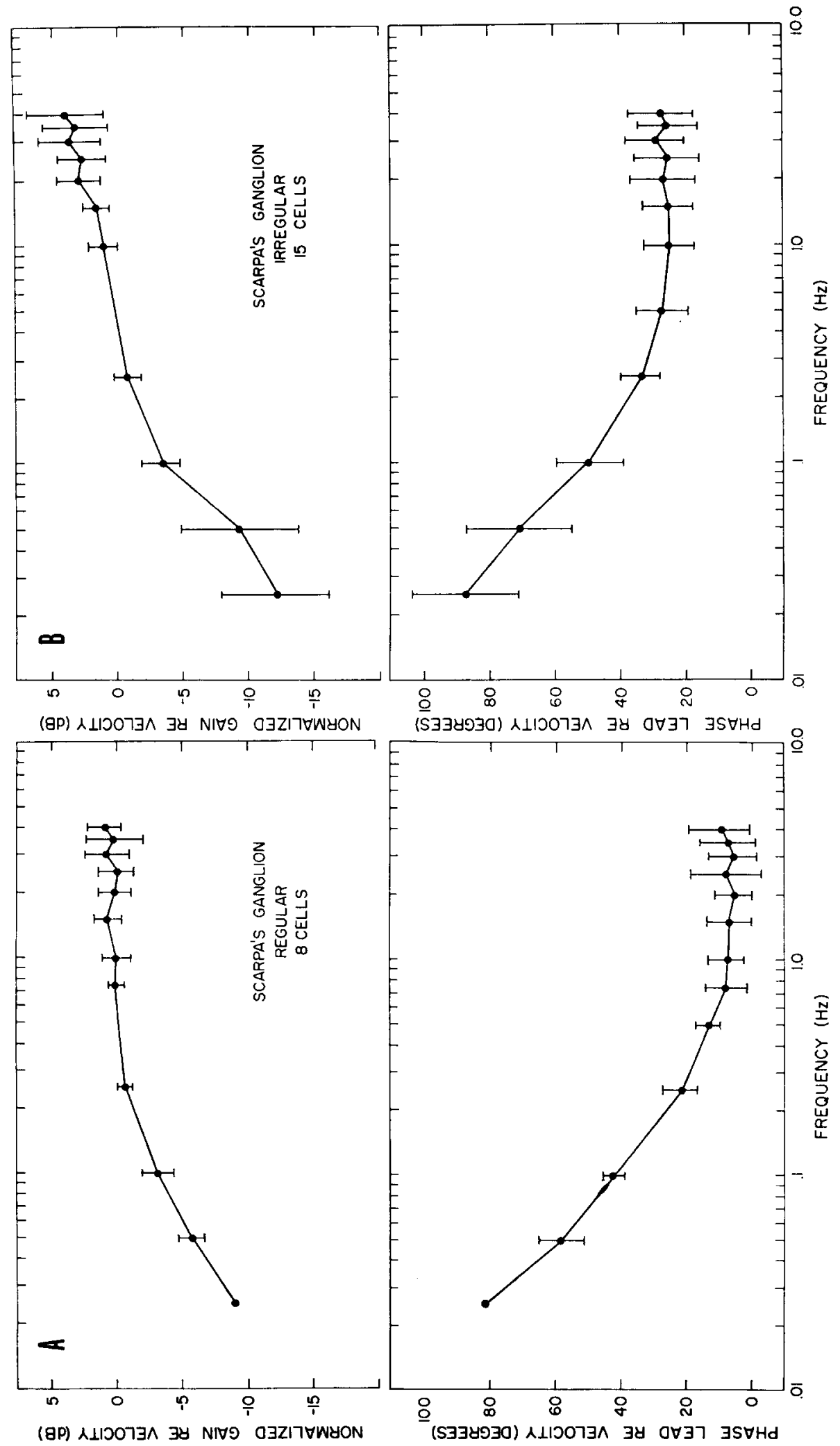

Fig. 5. Average and normalized Bode plots for 8 lateral canal regular Scarpa's ganglion cells in A and 15 lateral canal irregular Scarpa's ganglion cells in B. Brackets indicate population standard deviations. 
canal cells where the gain data of each cell were normalized by subtracting the gain in $\mathrm{dB}$ at $0.5 \mathrm{~Hz}$ before averaging the results. The standard deviation of the sample is indicated by the brackets around each point. The general features are those of a first order system as predicted by the theory for cupula movement. At frequencies above $0.5 \mathrm{~Hz}$ the gain is nearly constant while at lower frequencies it decreases with decreasing frequency. The phase of cell response is nearly in phase with angular velocity at high frequencies, and the phase lead increases with decreasing frequency below $1.0 \mathrm{~Hz}$, being nearly in phase with angular acceleration at $0.05 \mathrm{~Hz}$.

In comparison, Fig. 5B illustrates the average Bode plot for 15 irregular type lateral canal cells in Scarpa's ganglion. The features are considerably different from those of Fig. 5A. The phase curve is of similar shape but levels out at a much greater phase lead relative to angular velocity at high frequencies, and the gain at low frequencies has a slope greater than that of the regular cells. Furthermore, the gain continues to increase throughout the mid and high frequency regions with nearly constant slope.

Vestibular nuclei. Histology results showed most vestibular nuclei recordings to be in the medial vestibular nucleus and medial portions of the lateral vestibular nucleus. Fig. 6 shows the average Bode plot for 18 such cells. The features are similar

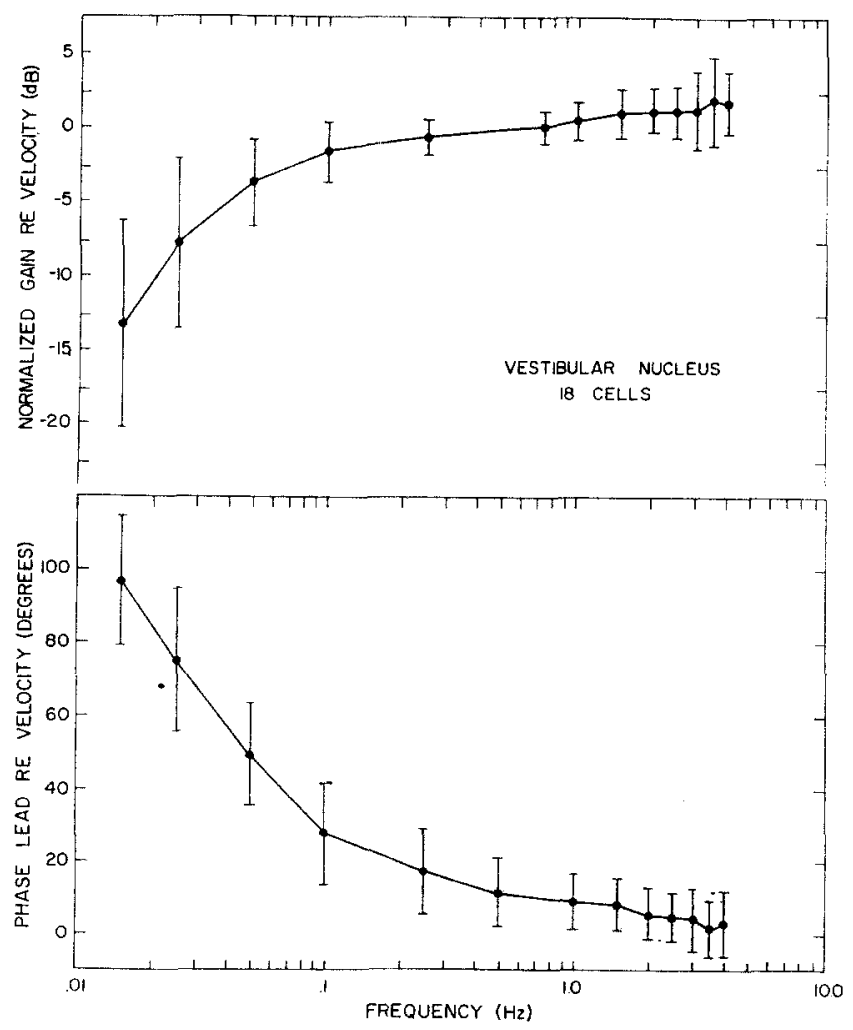

Fig. 6. Average and normalized Bode plot for 18 lateral canal type I cells in the vestibular nuclei. Brackets indicate population standard deviations. 
to those of the regular first order neurons. The phase is nearly in phase with angular velocity above $1.0 \mathrm{~Hz}$ and the gain is nearly flat in the mid and high frequency region. Of these 18 cells, 9 showed some skewness in the period histogram at the higher frequencies. This may explain the slight gain slope seen in this region, while the phase lead relative to angular velocity remains constant or even decreases.

The significant finding is that the second order neurons studied did not exhibit the phase lead characteristics of the irregular first order neurons. As pointed out previously, the effect of the phase synchronization of low discharging cells, if any, on the calculated phase angle is to increase the phase lead. Thus, the inclusion of some cells showing skewness in the period histogram has possibly biased the results toward showing a greater phase lead than is actually present at this level.

\section{Comparison of Bode plot results}

Fig. 7 compares the average Bode plot results for the 3 groups of cells. Functionally the important frequency range is probably above $0.5 \mathrm{~Hz}$. In this region, the second order cells and the regular cells are nearly in phase with head angular velocity and have a nearly constant gain. The irregular cells, however, show the large but constant phase lead with nearly constant gain slope. The mean phase lead for the 9 regular cells is $7.46^{\circ}$ (S.D. $=5.59$ ) while for the 15 irregular cells it is $26.29^{\circ}$ (S.D. $=$ 8.38). The mean phase lead for the vestibular nuclei cells if $6.38^{\circ}($ S.D. $=4.78)$ at 1.0 $\mathrm{Hz}$ but is seen in Fig. 7 to decrease even further as the frequency increases.

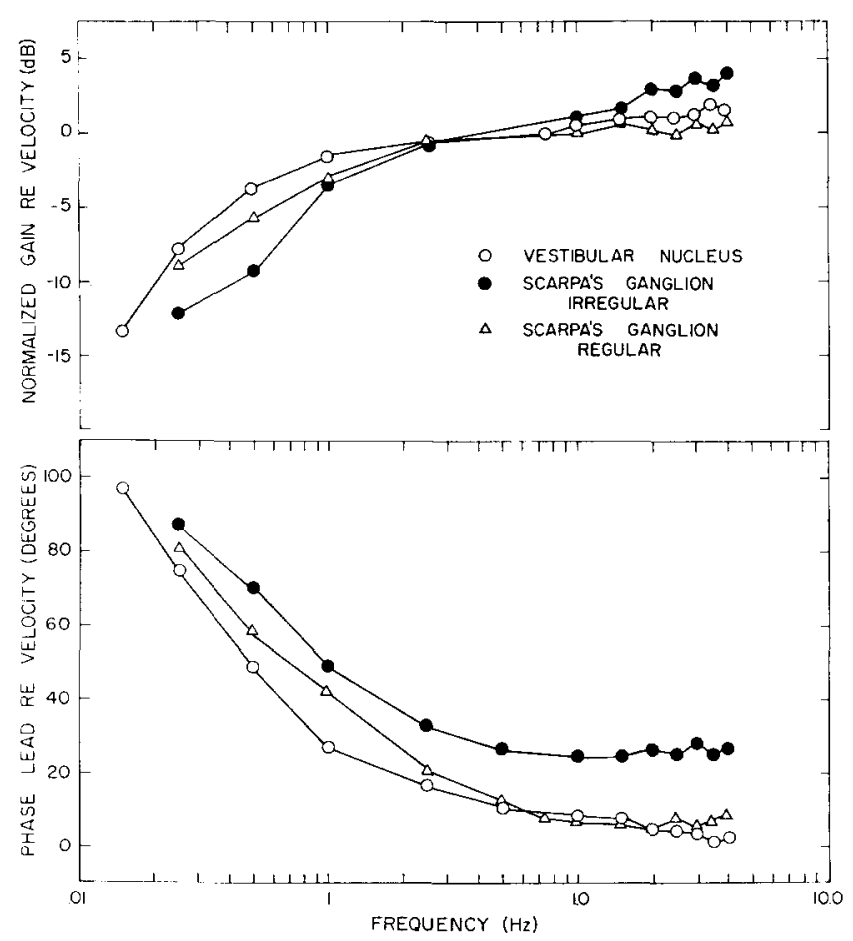

Fig. 7. Comparison of mean Bode plots for the 3 groups of vestibular cells -8 regular and 15 irregular first order neurons, and 18 second order neurons. 


\section{DISCUSSION}

\section{First order neurons}

In many respects the results of this study are in agreement with those of Fernández and Goldberg ${ }^{4}$ for the squirrel monkey. The regular neurons respond with transfer characteristics that can be accurately modeled by a linear first order system for frequencies less than $8 \mathrm{~Hz}$. These neurons show little or no adaptation, are nearly in phase with angular velocity, and have a constant gain above $1.0 \mathrm{~Hz}$. For the gerbil, an appropriate time constant appears to be between 2 and $3 \mathrm{sec}$ corresponding to a break frequency between 0.05 and 0.08 cycles/sec (i.e., the frequency at which the phase lead is $45^{\circ}$ ). This compares with a time constant of $4.0 \mathrm{sec}$ observed by Jones and Milsum $^{8}$ in the cat and $5.4 \mathrm{sec}$ observed by Fernández and Goldberg ${ }^{4}$ in the squirrel monkey. This faster time constant is consistent with the time constants for the cat and squirrel monkey when one considers the relation between the time constant and semicircular canal size determined by Jones and Spells? ${ }^{7}$. It was demonstrated that the time constant is proportional to the ratio $R^{2} / r^{2}$, where $R$ is the radius of curvature of the semicircular canal and $\mathrm{r}$ is the internal radius of the membranous duct. Using data from Igarash ${ }^{6}$ for the dimensions of the canals in the cat and squirrel monkey and from Jones and Spells ${ }^{7}$ for the dimensions of the canals in the jerboa mouse (which should be a good approximation to the gerbil), one obtains by the above relationship an estimate of $2-3.3 \mathrm{sec}$ for the time constant in the gerbil which agrees with the results of this study.

While the transfer characteristics of the irregular neurons in the gerbil are similar to that found by Fernández and Goldberg ${ }^{4}$ in that they deviate from a first order system at both low and high frequencies, the results of this study suggest a different transfer function. In both these studies, cells showed a tendency to adaptation. In the present study this phenomenon was observed using step accelerations of $40 \mathrm{sec}$ duration (2-6 degrees/sq.sec) on several occasions, and was also observed in the Bode plots of individua' cells as a phase lead relative to angular acceleration and greater than $20 \mathrm{~dB} /$ decade gain slope at low frequencies.

At higher frequencies Goldberg and Fernández observed a phase lead which reached a minimum between 0.5 and $1.0 \mathrm{~Hz}$ and continued to increase above $1.0 \mathrm{~Hz}$ while the gain showed a plateau between 0.5 and $1.0 \mathrm{~Hz}$ and then began to increase above $1.0 \mathrm{~Hz}$. These data were appropriately modeled by a zero in the transfer function and it was suggested that this characteristic could be due to a sensitivity of the neuron to cupula velocity as well as cupula position. In contrast, the results for the gerbil show a steady or constant gain slope (increasing with increasing frequency) from $0.1 \mathrm{~Hz}$ and up, and a constant but substantial phase lead above $0.5 \mathrm{~Hz}$. These characteristics suggest an alternative transfer function known as the fractional power law function. In Laplace notation this is written as $\mathrm{s}^{\mathrm{k}}$, where $\mathrm{k}$ is a fraction less than 1.0. In the time domain, this function multiplied by the gamma function $[\Gamma(1-\mathrm{k})]$ becomes $\mathrm{t}^{-\mathrm{k}}$ which describes the phenomenon of adaptation observed in the irregular cells.

This fractional power transfer function or fractional differentiator, as it is often 


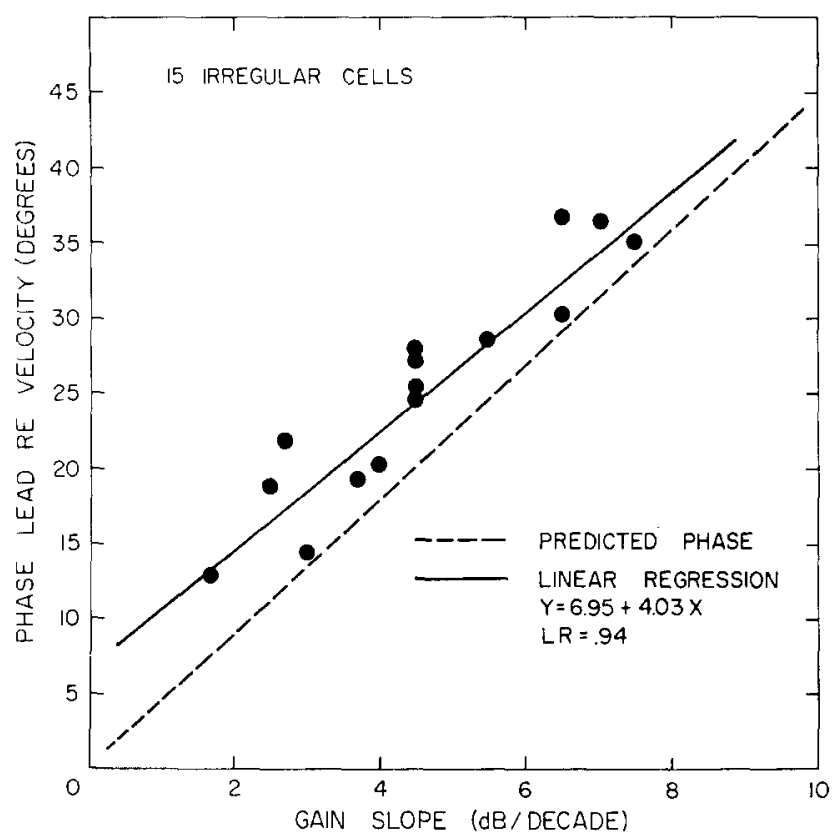

Fig. 8. Phase lead re angular velocity at $1.0 \mathrm{~Hz}$ versus gain slope of Bode plot between 0.4 and 4.0 $\mathrm{Hz}$ for 15 irregular cells. Solid line is linear regression fit to data. Broken line shows theoretical phasegain slope relation based on the fractional power transfer function.

called, has been observed by others ${ }^{1,2,9}$ in other mechanical biological transducers. It predicts a constant phase shift in the Bode plot of $k$ times $90^{\circ}$. Fig. 8 shows the results of plotting the gain slope between 0.4 and $4.0 \mathrm{~Hz}$ versus the phase lead at $1.0 \mathrm{~Hz}$ for the 15 irregular cells. It is seen that there is an excellent linear relation $(R=0.94)$ between these two measures, and that this agrees well with the theoretical prediction of the fractional power law transfer function shown by the broken line. While the power law function does not account for all the phase lead based upon the gain slope measure, it is interesting that the difference is about equal to the phase lead of the tegular cells $\left(7.46^{\circ}\right)$ and may be accounted for by other system dynamics.

For the 15 irregular cells in this study, the mean phase lead at $1.0 \mathrm{~Hz}$ was $26.29^{\circ}$. This corresponds to an average $\mathrm{k}$ value of about 0.29 . The largest phase lead observed at $1.0 \mathrm{~Hz}$ was $37^{\circ}$ corresponding to a $\mathrm{k}$ of 0.41 .

Transfer function for Scarpa's ganglion cells. For the regular first order neurons the torsion pendulum model can be used to describe the transfer characteristics. While this can be simply modeled by a first order system with a single time constant for the frequency range of interest, the system is classically written as a second order system involving two time constants. One time constant is the ratio of the viscous damping couple to the elastic restoring couple $(\pi / \Delta)$ of the cupula-endolymph system and is the constant determined in this study to be about $2.5 \mathrm{sec}$ for the gerbil. The second time constant is the ratio of the moment of inertia of the cupula-endolymph system to the viscous damping couple $(\theta / \pi)$ and may be estimated from hydrodynamic calculations. For the gerbil it is approximately $2 \mathrm{msec}$. An appropriate 
transfer function for the regular first order neurons in gerbil may therefore be written as:

$$
H(S)=\frac{\text { Neuron firing rate }}{\text { Angular velocity }}=\frac{s}{(1+2.5 s)(1+0.002 \mathrm{~s})}
$$

For the irregular neurons the fractional power law function must be included giving:

$$
\mathrm{H}(\mathrm{S})=\frac{T(1-\mathrm{k}) \mathrm{s}^{\mathrm{k}+1}}{(1+2.5 \mathrm{~s})(1+0.002 \mathrm{~s})} ; 0<\mathrm{k}<0.5
$$

Since $(1-k) s^{k}$ approaches 1 as $k$ approaches zero, the above function can be used to describe the transfer function for all Scarpa's ganglion neurons, where the special case of $\mathrm{k}=0$ applies to the regular type neurons.

Vestibular nuclei. Both Jones and Milsum ${ }^{8}$ and Shinoda and Yoshida ${ }^{11}$ have determined transfer characteristics of vestibular nuclei neurons in the cat, but neither used stimulation frequencies above $1.0 \mathrm{~Hz}$. Jones and Milsum found the transfer characteristics for the second order neurons to be similar to the first order system in this frequency range. Shinoda and Yoshida indicated that there were possibly two groups of neurons, one which was in phase with angular velocity above $0.2 \mathrm{~Hz}$ and the other showing about $30^{\circ}$ phase lead in this region. The results of the present study indicate no evidence of vestibular nuclei neurons with large phase leads at higher frequencies and suggest that at this level the frequency components of vestibular signals between 0.5 and $5 \mathrm{~Hz}$ are in phase with head angular velocity and have a constant gain. With respect to the vestibulo-ocular reflex, then, a second integration between the nuclei and the eye muscles is appropriate to produce signals proportional to head position.

In a few cases, the responses to step accelerations $\left(2-6^{\circ} / \mathrm{sq} . \mathrm{sec}\right.$ for $\left.40 \mathrm{sec}\right)$ were obtained for vestibular nuclei neurons. These responses indicated varying degrees of adaptation even though the Bode plots showed little evidence of phase lead at frequencies to $5.0 \mathrm{~Hz}$. As with the irregular first order neurons, this adaptation shows in the Bode plot as a greater phase lead and decreased gain at low frequencies from that expected for a first order system (see Fig. 6). The fact that this adaptation occurs without the phase lead at higher frequencies, suggest that its presence at this level may be due to a different mechanism than in the first order irregular neurons.

\section{Significance of fractional power transfer function}

From a physiological standpoint, it is difficult to give physical meaning to a fractional power transfer function. Several models for its generation in other receptor systems have been proposed by others. These include, a power law spring, diffusion properties of transmission lines, and 'Gaussian distribution about a simple average rate constant' 3 . A more recent model by Thorson and Biederman-Thorson ${ }^{12}$ which suggests that "one might simply treat the power law behavior as a weighted summation over a broad range of simple exponential relaxation processes' is perhaps the most plausible. In this distributed parameter system the transfer function for each step response, $e^{-a t}$, is that of a high pass filter $s /(s+a)$, and each makes a contribution 
which is proportional to its own rate constant raised to the power $(\mathrm{k}-1) \cdot \mathrm{s}^{\mathrm{k}}$ can then be represented as a sum over a continuum of high-pass filters with the same weighting function.

$$
\mathrm{s}^{\mathrm{k}}=\frac{1}{\Gamma(\mathrm{k}) \Gamma(1-\mathrm{k})} \int_{0}^{\infty} \mathrm{a}^{\mathrm{k}-1} \frac{\mathrm{s}}{\mathrm{s}+\mathrm{a}} \mathrm{da}
$$

Where such a distributed process exisis in the vestibular system (or any receptor system) can only be conjectured at this time. One possibility is in the electrical processes involved in impulse initiation in the primary neuron. The distributed process could result from the ramifications of a single neuron to many sensory hair cells. This hypothesis, however, suggests that the irregular cells should innervate many sensory cells and is in opposition to current anatomical and physiological evidence. Wersäll14 has observed that the thin first order fibers ramify profusely in the subepithelial space and innervate many type II sensory cells on the sides of the cristae. The thick fibers seem to go to the central or top regions of the cristae and innervate only a few type I hair cells. Fernández et al. ${ }^{5}$ and Walsh et al. ${ }^{13}$ have observed that irregular cells are most easily found in regions of Scarpa's ganglion composed of a majority of thick fibers, and regular cells are found in regions containing mostly thin fibers.

An alternative possibility is that the distributed mechanism is in the processes involved at the sensory cell itself, the generator potential being the summation of the processes. Such a mechanism may be electrical, chemical, or even mechanical in nature.

Whatever the mechanism, the results from the small sample of second order neurons in this study suggest that these lead compensation characteristics of the irregular neurons do not carry over to the second order neurons in the nucleus. It is possible that the irregular neurons project to regions of the vestibular nuclei not recorded from in this study. It may also be the case that the lead characteristics are removed by a low pass filter property of the synaptic process in the vestibular nuclei.

\section{Effect of anesthesia}

In any study involving the responses of neurons more central than the first order sensory neurons, the effect of anesthetics is always a concern. Thus, the possibility that the anesthetics used may have influenced the responses of second order neurons must be considered in this study. Precautions were taken, however, to minimize these influences.

For one thing, non-barbiturate anesthetics were used. An initial dose of urethane was given for the surgery and the animals were maintained on a minimum of Ketalar for the remainder of the experiment. Also, once the surgery was completed the animal was normally allowed to rest for $6-8 \mathrm{~h}$ before recordings were attempted. Experiments with urethane on unoperated animals indicated that the major effects of the urethane were gone by this time. In this regard, it is also significant that the average neural gain and resting discharge level for second order neurons in this study are in excellent agreement with values found by Jones and Milsum ${ }^{8}$ in the unanesthetized (decerebrate) cat. 


\section{ACKNOWLEDGEMENTS}

The authors gratefully acknowledge the advice and assistance of Drs. N. B. Gross, Jack H. Young, and Wladimiro Lifschitz. We also wish to express appreciation to the technical staff of the Kresge Hearing Research Institute, University of Michigan.

This study was supported by a grant from the John A. Hartford Foundation. Program Project Grant NS-05785, and N.I.H. Grant NS-10854.

\section{REFERENCES}

1 Brown, M. C. and Stein, R. B., Quantitative studies on the slowly adapting stretch receptor of the crayfish, Kybernetik, 3 (1966) 175-184.

2 Chapman, K. M. and Smith, R. S., A linear transfer function underlying impulse frequency modulation in a cockroach mechanoreceptor, Nature (Lond.), 197 (1973) 699-700.

3 Cole, K. S. and Cole, R. H., Dispersion and absorption in dielectrics. I. Alternating current characteristics, J. chem. Physiol., 9 (1941) 341-351.

4 Fernández, C. and Goldberg, J. M., Physiology of peripheral neurons innervating semicircular canals of the squirrel monkey. II. Responses to sinusoidal stimulation and dynamics of peripheral vestibular system, J. Neurophysiol., 34 (1971) 661-675.

5 Fernández, C., Goldberg, J. M. and Abend, W. K., Response to static tilts of peripheral neurons innervating otolith organs of the squirrel monkey, $J$. Neurophysiol., 35 (1972) 978-997.

6 Igarashi, M., Dimensional study of the vestibular end organ apparatus. In J. Huertas (Ed.), Second Symposium of the Role of the Vestibular Organ in Space Exploration, NASA Sp-115, Washington, D. C., 1966, pp. 46-54.

7 Jones, G. Melville and Spells, K. E., A theoretical and comparative study of the functional dependence of the semicircular canal upon its physical dimensions, Proc. roy. Soc. B, 157 (1963) 403-419.

8 Jones, G. M. and Milsum, J. H., Frequency response analysis of central vestibular unit activity resulting from rotational stimulation of the semicircular canals, J. Physiol. (Lond.), 219 (1971) 191-215.

9 McCall, W. C., Jr., Dynamic Response of Slowly Adapting Cat Knee Joint Receptors, Ph.D. Thesis, Univ. of Michigan, Ann. Arbor, 1971.

10 Oman, C. M. and Young, L. R., The physiological range of pressure difference and cupula deflections in the human semicircular canal, Acta otolaryng. (Stockh.), 74 (1972) 324-331.

11 Shinoda, Y. and Yoshida, K., Dynamic characteristics of responses to horizontal head angular acceleration in vestibulo-ocular pathway in the cat, $J$. Neurophysiol., 37 (1974) 653-673.

12 Thorson, J. and Biederman-Thorson, M., Distributed relaxation processes in sensory adaptation, Science, 183 (1974) 161-172.

13 Walsh, B. T., Millers, J. B. Gacek, R. R. and Kiang, N. Y. S., Spontaneous activity in the eighth cranial nerve of the cat, Int. J. Neurosci., 3 (1972) 221-235.

14 Wersäll, J., Studies on the structure and innervation of the sensory epithelium of the cristae ampullae in the guinea pig. A light and electron microscopic investigation, Acta otolaryng. (Stockh.), Suppl. 126 (1956).

15 Young, J. H., Analysis of Vestibular System Responses to Thermal Gradients Induced in the Temporal Bone, Ph.D. Thesis, Univ. of Michigan, Ann Arbor, 1972. 